\title{
On the origin of comet C/1999 S4 LINEAR
}

\author{
C. de la Fuente Marcos and R. de la Fuente Marcos
}

Universidad Complutense de Madrid, Ciudad Universitaria, 28040 Madrid, Spain

Received 20 August 2001 / Accepted 3 September 2002

\begin{abstract}
Current models of the formation of comets in the Solar System assume that the most likely formation site for these pristine objects is in the Uranus-Neptune zone or just beyond with subsequent dynamical ejection by the growing protoplanets to distant orbits to form the Oort cloud. However, the composition of the recently disintegrated comet C/1999 S4 LINEAR suggests that it was most likely formed in the Jupiter-Saturn region (Mumma et al. 2001b; Kawakita et al. 2001). In this paper we argue that cometesimals could easily appear inside exterior resonances resulting from gas drag, between the orbits of Jupiter and Saturn and beyond Saturn, during the formation of these giant planets. Then, the trapped material could evolve into actual comets by means of collisional coagulation, followed by gravitational instability of a layer of macroscopic bodies or two-body accretion. Properties of these objects would be rather different from those found in classical comets formed beyond Neptune as a result of different physicochemical environment. Our results also suggest that temporarily stable cometary belts may have existed in the Jupiter-Saturn region. We also discuss the implications of this scenario on the existence of a bimodal Centaur population.
\end{abstract}

Key words. minor planets, asteroids - comets: general - comets: individual: C/1994 S4 LINEAR - Kuiper belt - Oort cloud solar system: formation

\section{Introduction}

Theories of cometary origin fall into two groups: primordial theories in which comets formed concurrently with the Sun and planets and have been stored in the Oort cloud over the age of the Solar System, and episodic theories in which comets either formed in distant interstellar clouds and were subsequently captured by the Solar System or comets formed as a result of catastrophic, recent and possibly repeated events in the planetary region (Mumma et al. 1993; Irvine et al. 2000). Current observational findings favour primordial theories; in this scenario, comets scattered outwards gave rise to the Oort cloud and comets scattered inwards contributed to the heavy bombardment in the inner Solar System and may have brought the ocean water and pre-biotic organic material to Earth.

A widely accepted model of the formation of comets in the Solar System assumes that most long-period comets originated in the Uranus-Neptune zone or just beyond with subsequent dynamical ejection by the growing protoplanets to distant orbits to form the Oort cloud (see, e.g., Duncan et al. 1987 and Stern \& Weissman 2001 for details). However, if comets, and possibly some other minor bodies, were formed soon after the first planet-sized objects (likely proto-Jupiter and protoSaturn), they should appear in a region not too close to the growing protoplanets as to allow temporarily steady dynamical evolution of the rocky material. Mumma et al. (2001a)

Send offprint requests to: C. de la Fuente Marcos, e-mail: nbplanet@ucmail.ucm.es have found that the measured compositions of four well studied comets (Halley, Hyakutake, Hale-Bopp and Lee) are consistent with formation from interstellar ices in the nebular region beyond Uranus. Nevertheless, the unusual organic composition of comet C/1999 S4 LINEAR suggests that it was formed in the inner part of the outer disk of the Solar System, most likely in the Jupiter-Saturn region (Mumma et al. 2001b) as it was depleted in carbon-chain molecules relative to most other comets. In addition, the chemistry of the building blocks of the nucleus was very uniform (Bockelée-Morvan et al. 2001) and this may thus also apply to their formation region between Jupiter and Saturn in the protoplanetary disk. Recently, Kawakita et al. (2001) have collected high-resolution spectra from the Subaru telescope of this object to determine the ortho-to-para ratio of $\mathrm{NH}_{2}$. By assuming that the $\mathrm{NH}_{2}$ is derived from the photodissociation of ammonia by the solar radiation, they derived a spin temperature of the ammonia of about $38 \mathrm{~K}$. If this ammonia is primordial, then the spin temperature indicates that the comet formed between 8 and $15 \mathrm{AU}$ in the solar nebula. Peculiar objects with unusual organic composition, tentatively identified as formed in the Jupiter-Saturn nebular region, have been named Jovian-class comets (Mumma et al. 2001b), although they should not be confused with the Jupiter-family comets, a dynamical grouping with Tisserand invariant in the span 2-3 and orbital periods $<20$ yr that formed over a much wider range of heliocentric distance and only later entered their present orbits (Fernández et al. 1999). In a very recent paper, Nurmi et al. (2002) conclude that the Jupiter-family comets 
cannot originate in the spherically distributed Oort Cloud and that the existence of this dynamical class can be explained only if they are captured from the extended disk of comets with semimajor axes $a<5000$ AU. Typical members of the Jupiter-family are the comets 9P/Tempel 1 and 19P/Borelly (Soderblom et al. 2002).

C/1999 S4 LINEAR was a unique object in many different aspects (Mäkinen et al. 2001; Mumma et al. 2001b; Weaver et al. 2001; Kawakita et al. 2001). This peculiar comet was discovered on 27 September 1999 by the Lincoln Near Earth Asteroid Research (LINEAR) program, while still at 4.2 AU from the Sun. The comet became a favorable target for observations during July 2000. It passed its closest point to Earth, 0.364 AU, on 23 July 2000, 3 days before it reached perihelion. Surprisingly, the comet completely disintegrated within a few days of its closest approach to Earth. Photometric measurements (Farnham et al. 2001) suggest a lower limit of $0.44 \mathrm{~km}$ for the radius of the cometary nucleus before breakup. Photometric analysis (Weaver et al. 2001) of the fragments indicates that the largest ones have effective spherical diameters of about $100 \mathrm{~m}$. The total mass detectable in the dust tail after the breakup was $3 \times 10^{8} \mathrm{~kg}$ suggesting that most of the comet's original mass after breakup was in the form of remnants between $1 \mathrm{~mm}$ and $50 \mathrm{~m}$ in diameter. Both its orbit and activity evolution during its approach to perihelion suggest that C/1999 S4 LINEAR was a dynamically new comet from the Oort cloud, possibly on one of its first close visits to the Sun (Farnham et al. 2001).

In this paper we suggest a plausible mechanism for the formation of Jovian-class comets: outer resonance trapping resulting from gas drag (Weidenschilling \& Davis 1985; Patterson 1987; Beaugé \& Ferraz-Mello 1993; Kary et al. 1993; Scholl et al. 1993; Beaugé et al. 1994). We model the evolution of solid particles subject to nebular gas drag and perturbation from growing protoplanets (Jupiter and Saturn). Particles of various masses are distributed on almost circular coplanar orbits external to the protoplanets. As their orbits decay sun-ward, solid particles become trapped in external gas-induced mean-motion resonances with the protoplanets. While in theory this trapping may be permanent, trapping lifetime is limited by several mechanisms, such as growth of the protoplanets, formation of Uranus and Neptune and dispersal of the protoplanetary nebula. We begin in Sect. 2 by introducing the basic concepts considered in this paper. We analyze the results of our calculations in Sect. 3. In a concluding section a discussion of the implications of these results is presented.

\section{Outer resonance trapping from gas drag}

Orbits of dust grains in a gaseous protoplanetary nebula are not stable due to gas drag. Both orbital eccentricity and inclination are damped, although the most important effect is the secular decrease of the semi-major axis. Under the standard assumptions (axisymmetric nebula), the gas flows around the Sun at slightly less than the circular Keplerian speed and the solid particles are subject to a systematic drag force which makes them drift radially inwards (Weidenschilling 1977); this is because the gas is pressure supported and the particles are not.
The difference between the angular speed of the gas and the Kepler frequency is: $\Delta \Omega=-\xi \Omega(r)$, where $\xi$ is a small factor which accounts for the negative radial pressure gradient (Adachi et al. 1976). This small perturbation causes the solid particles to experience a loss of energy and angular momentum, even if considering circular orbits. However, solid (metric) particles approaching a growing protoplanet in the presence of (weak) gas drag can become trapped in mean-motion resonances (Weidenschilling \& Davis 1985), at which point their orbital migration effectively stops, and their eccentricities reach an equilibrium between resonance pumping and drag-induced damping. As a solid particle approaches a resonance, perturbations from the planet can excite the particle's eccentricity and increase its semi-major axis. If the drag force is not very strong, these resonant perturbations will be able to compensate for the particle's orbital evolution, and the particle will evolve into a stable equilibrium orbit determined by the relative strengths of the drag and the resonance. Therefore, in an outer resonance where a particle revolves slower than a planet or planets, gain and loss of orbital momentum as well as of orbital energy may be kept in equilibrium. The time scale for this equilibrium state depends on the values for the planetary masses, for the eccentricity of the planetary orbits, for the particles' radii and on the order of the resonance. These values can be determined by numerical simulations. Resonant capture can produce cometary belts beyond Saturn but also between Jupiter and Saturn (Lecar et al. 1992). Resonances discussed in this paper are not classical gravitational resonances but gasdriven gravitational ones. The cosmogonic implications (formation of the outer planets) of this process have been mainly considered by Patterson (1987) and Beaugé et al. (1994) although little consideration has been given to the role of this mechanism in the formation of minor bodies in the outer Solar System. The most remarkable cosmogonic result in this field is the conclusion (Beaugé et al. 1994) that the formation mechanism originally proposed by Patterson (1987) is plausible and may well explain the quasi-resonant configuration of the actual outer planets.

The phenomenon of exterior resonance trapping induced by gas drag has been studied from a strictly theoretical point of view in the framework of the planar restricted three-body problem (star, protoplanet and solid particle). The first analysis was made by its discoverers, Weidenschilling \& Davis (1985), and they concluded that trapping is only possible when the falling body experiences an exterior commensurability relation with the perturbing protoplanet. In this scenario, the secular and simultaneous decrease in both the semi-major axis and eccentricity of the falling body induced by the drag force is balanced by an equally secular and simultaneous increase in the same orbital elements driven by the gravitational contribution of the protoplanet. A detailed analysis of this process has been carried out by Beaugé \& Ferraz-Mello (1993), finding the conditions for trapping, as well as the final stable orbits of the falling bodies. Although in our present work we consider the more general case of two growing protoplanets but still coplanar, we will now summarize theoretical results relevant for our current study from Beaugé \& Ferraz-Mello (1993) as well as numerical results from Beaugé et al. (1994). If we assume that the ratio 
between the orbital periods of the protoplanet and the falling body lies close to a generic $(p+q) / p$ ( $p$ and $q$ are small integers with $p<0$ ) exterior mean-motion resonance:

(a) For a given resonance, trapping can occur only for particles larger than a critical size. In general, larger bodies will be preferentially trapped at distant resonances. Therefore, exterior resonance trapping resulting from gas drag is able to induce mass segregation in an evolving protoplanetary disk.

(b) This trapping mechanism is extremely sensitive to the initial conditions. Falling from outside a certain resonance and having a size less than the critical for capture does not ensure that capture indeed occurs as initial orbital elements play a key role in the trapping process. The object may cross that given resonance and become trapped by another one with smaller radial distance.

(c) The final orbits of the trapped bodies are of two types: $\sigma$-libration and corotation. For $\sigma$-libration orbits, accumulation occurs only in the radial direction but not in the azimuthal angle and the eccentricity changes in the range $0.03-0.12$. In corotation orbits, coherent motions appear with particles being clustered about $|p|$ evenly spaced phases of the azimuthal angle and the eccentricity is a fixed universal quantity, depending only on the order of the resonance.

(d) There is a rivalry among different resonances and no particular commensurability dominate the rest and control the accretion and dynamics of the system.

In our calculations, we investigate a model composed of the Sun, an existing proto-Jupiter (and also proto-Saturn) and a planar extended ring of solid bodies embedded in the primordial solar nebula. Our model neglects two-body interactions between solid bodies. By including the combined effects of gravitational perturbations and gas drag we have simulated the orbital evolution of primordial solid material and obtained some insight into the role that gas-induced resonance trapping could have played in minor body formation at the JupiterSaturn region. These minor bodies include the Jovian-class comets, in particular C/1999 S4 LINEAR, and possibly some Centaurean objects. If only proto-Jupiter is included, our numerical results should match those from the theory (Beaugé \& Ferraz-Mello 1993) and numerical experiments (Beaugé et al. 1994). However, results from models including two protoplanets are presented here for the first time.

\section{Modeling and results}

In order to study whether or not resonance trapping of particles is a plausible mechanism to confine solid material able to form primordial comets we consider the epoch when the masses of proto-Jupiter and proto-Saturn reach the present values by accreting the gases of the solar nebula. During this stage, draginduced orbital decay of material from the outer solar nebula provides continuous replenishment of solid particles. We have carried out a series of numerical integrations of coplanar orbits of solid metric particles in the gravitational field of the Sun, proto-Jupiter and proto-Saturn, using the BulirschStoer method (Bulirsch \& Stoer 1966). We investigate the response of the particles, in a standard protoplanetary (see, e.g., Cuzzi et al. 1993) disk with radius $R_{\mathrm{D}}=100 \mathrm{AU}$ and mass
$M_{\mathrm{D}}=0.001 M_{\odot}$ (as Jupiter and Saturn are being formed). Our parallel direct integration code models gravitational and viscous forces, including self-gravitation (de la Fuente Marcos $\&$ de la Fuente Marcos 1998, 2001). We decide to focus our attention in meter-sized particles following Weidenschilling (1994). In his work he argues that cometary and Chiron-like objects could form by accretion of primary nuclei or building blocks, tens of metres in size. This theory is consistent with the observed sizes and structure of comet nuclei as well as outbursts and splitting events. On the other hand, photometric observations of the debris left by the disintegration of C/1999 S4 LINEAR suggest that the primordial building blocks of this comet were indeed metric in size, therefore metric (or submetric) particles are the most relevant as regards comet formation. In the classical core-instability scenario of giant planet formation (see, for example, Mizuno 1980), when the proto-giant planets were already formed, the planetesimals have grown to kilometre-sized bodies, therefore there is little room to comet formation following the building blocks model pointed out above. However, in the framework of the capturein-vortex mechanism (see, for example, Barge \& Sommeria 1995; Godon \& Livio 2000; de la Fuente Marcos \& Barge 2001; de la Fuente Marcos et al. 2002) the birth of the Giant planets could take less than a million years as well as allow for coexistence of metric particles and proto-giant planets. In the following, we will assume this non-standard scenario.

In our calculations, the initially circular coplanar orbits of the test particles are perturbed by the Sun, proto-Jupiter and proto-Saturn but do not themselves exert any gravitational forces. The initial heliocentric distance of the dust particle population is $20 \mathrm{AU}$. For the protoplanets we adopted their present eccentricities. Our models are followed for about $7 \mathrm{Myr}$ as recent determinations constrained by D/H measurements in the Solar System (Hersant et al. 2001) suggest that the epochs of the formation of Jupiter and Saturn cannot be lower than 0.7 and 5.7 Myr, respectively, after the formation of the Sun. Instead of considering a continuous growth model for the giant planets we restrict our calculations to several static combinations for the masses of the growing protoplanets: $M_{\mathrm{pJ}}$, $M_{\mathrm{pS}}=\left(M_{\mathrm{J}} / 10,0\right),\left(M_{\mathrm{J}}, 0\right),\left(M_{\mathrm{J}} / 10, M_{\mathrm{S}} / 100\right),\left(M_{\mathrm{J}}, M_{\mathrm{S}} / 10\right)$, $\left(M_{\mathrm{J}}, M_{\mathrm{S}}\right)$, where $M_{\mathrm{pJ}}$ is the mass of proto-Jupiter, $M_{\mathrm{pS}}$ is the mass of proto-Saturn and $M_{\mathrm{J}}$ and $M_{\mathrm{S}}$ are the masses of Jupiter $\left(0.000956 M_{\odot}\right)$ and Saturn $\left(0.000286 M_{\odot}\right)$, respectively. Our qualitative results do not strongly depend on the choice of protoplanetary masses but the quantitative results (order of resonances) depend slightly on these values (see Table 1).

Our first calculation considers a proto-Jupiter with $M_{\mathrm{pJ}}=$ $0.1 M_{\mathrm{J}}$ orbiting around the Sun with its current values of semimajor axis and eccentricity. Metric particles are trapped in outer resonances with semi-major axis 7.2 AU and eccentricity changing in the range [0.03, 0.09] with a period of about $15000 \mathrm{yr}$. Increasing the mass of the growing proto-Jupiter shifts the non-gravitational resonance outwards. For a $M_{\mathrm{pJ}}=$ $M_{\mathrm{J}}$ resonant capture for metric particles appears at 8.7 AU. The eccentricity is now changing in the interval [0.05, 0.12] with a period of a few $10^{3} \mathrm{yr}$. The exact values depend on the size of the particle: larger radius implies higher eccentricity and longer period. Besides the 8.7 AU resonance we find another one (see 
Table 1. Average orbital elements of the trapped bodies for the numerical experiments performed.

\begin{tabular}{llllc}
\hline \hline Size & $M_{\mathrm{pJ}}^{\star}$ & $M_{\mathrm{pS}}^{\star \star}$ & $a^{\star \star \star}$ & $e$ \\
\hline metric & 0.1 & 0.0 & 7.2 & {$[0.03,0.09]$} \\
metric & 1.0 & 0.0 & 8.7 & {$[0.05,0.12]$} \\
& 1.0 & 0.0 & 11.4 & {$[0.10,0.16]$} \\
submetric & 0.1 & 0.01 & 6.8 & {$[0.03,0.07]$} \\
metric & 0.1 & 0.01 & 16.1 & {$[0.10,0.15]$} \\
metric & 1.0 & 0.1 & 12.5 & {$[0.05,0.12]$} \\
centimetric & 1.0 & 1.0 & 12.5 & {$[0.08,0.16]$} \\
decimetric-metric & 1.0 & 1.0 & 13.3 & {$[0.04,0.12]$} \\
metric & 1.0 & 1.0 & 16.1 & {$[0.10,0.16]$} \\
& 1.0 & 1.0 & 17.1 & {$[0.22,0.23]$} \\
& 1.0 & 1.0 & 17.5 & {$[0.14,0.16]$} \\
& 1.0 & 1.0 & 17.9 & {$[0.10,0.12]$} \\
& 1.0 & 1.0 & 18.8 & {$[0.12,0.16]$} \\
\hline
\end{tabular}

${ }^{\star}$ proto-Jupiter mass (in $\left.M_{\mathrm{J}}\right)$.

${ }^{\star \star}$ proto-Saturn mass (in $M_{\mathrm{S}}$ ).

$\star \star \star$ semi-major axis (in $\mathrm{AU}$ ).

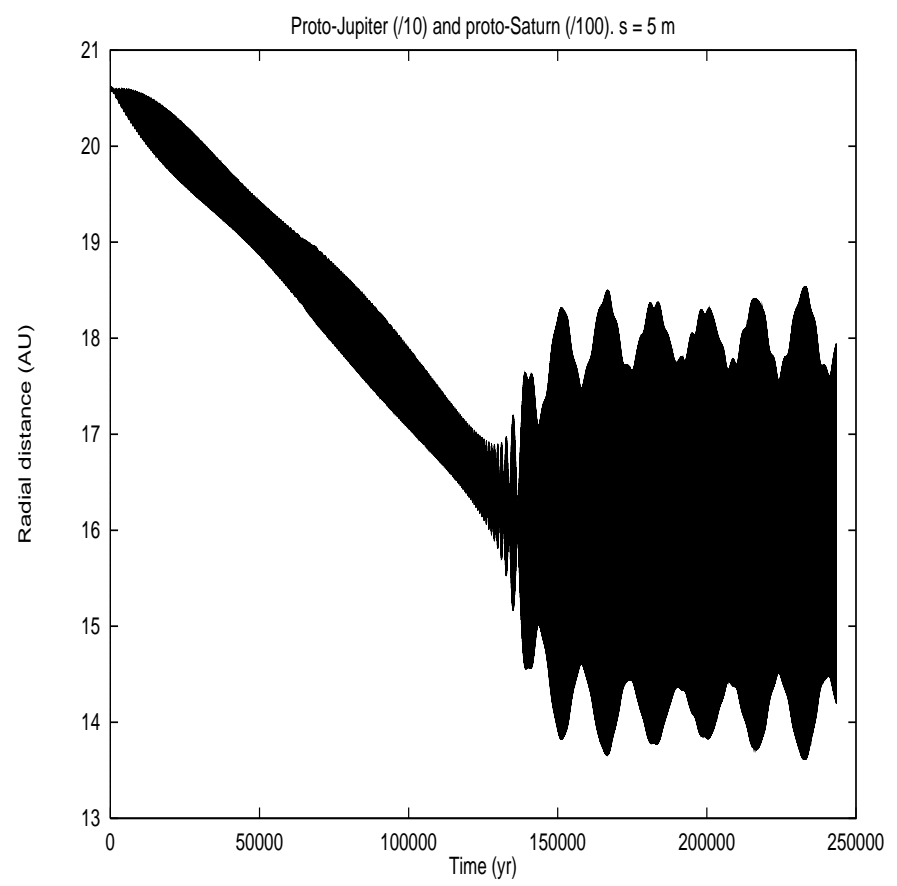

Fig. 1. Resonant trapping of a $5 \mathrm{~m}$ particle by proto-Jupiter $\left(M_{\mathrm{J}} / 10\right)$ and proto-Saturn $\left(M_{\mathrm{S}} / 100\right)$.

Table 1 and Fig. 3) at about 11.4 AU with higher eccentricity, span $0.10-0.16$. This outer resonance is stronger as shown in Fig. 4 (lower panel). Figure 4 provides another characteristic of gas-induced resonance trapping, size-selective capture. The outermost resonance is rather size-selective as the innermost shows clear size gaps.

The inclusion of a growing proto-Saturn changes the results dramatically. If $M_{\mathrm{pJ}}=0.1 M_{\mathrm{J}}$ and $M_{\mathrm{pS}}=0.01 M_{\mathrm{S}}$, particles of about $1 \mathrm{~m}$ or less are not captured by the outer resonances

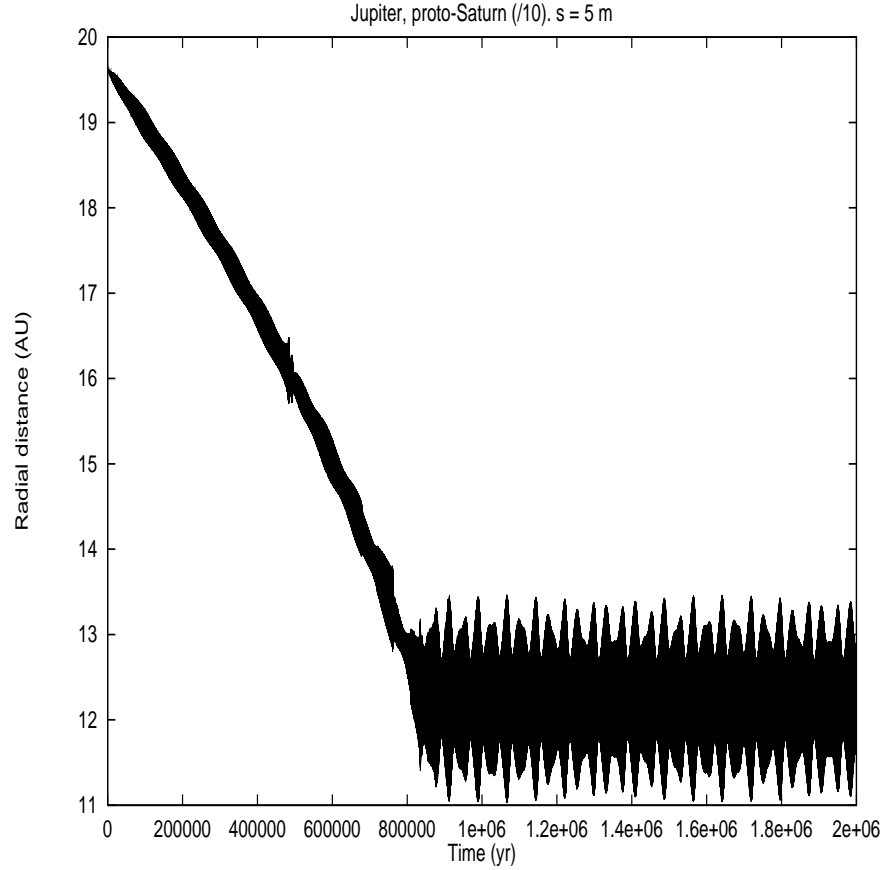

Fig. 2. Resonant trapping of a $5 \mathrm{~m}$ particle by Jupiter and proto-Saturn $\left(M_{\mathrm{S}} / 10\right)$.

beyond Saturn but cross the Saturnian orbit and stop at $6.8 \mathrm{AU}$ from the Sun. However, particles of about $5 \mathrm{~m}$ are trapped in an outer resonance at $16 \mathrm{AU}$ with the eccentricity in the range [0.100, 0.153] in a time scale of $12500 \mathrm{yr}$ (see Fig. 1). This is the vicinity of the $2: 1$ resonance with Saturn. Increasing the proto-Jovian mass $M_{\mathrm{pJ}}=M_{\mathrm{J}}$ and the proto-Saturn mass, $M_{\mathrm{pS}}=0.1 M_{\mathrm{S}}$, shifts this non-gravitational resonance inwards, 12.5 AU (Fig. 2). This is the 3:2 resonance with Saturn. When we consider current values for the masses of both Jupiter and Saturn, the resonant configuration also changes. Centimetric particles are trapped at the 3:2 resonance but decimetric grains are trapped at 13.3 AU and metric particles at the 2:1 resonance. Metric particles (see Figs. 4 and 3) are also trapped by other resonances (see Table 1), in fact the two resonances that appear in the Jupiter-alone case have been shifted outwards. Again, the size-selective nature of the trapping mechanism is clearly seen on the plots. The innermost resonance only includes particles of about $21 \mathrm{~m}$ and several size gaps appear in Fig. 4.

In summary, our results show that, depending on the masses of the protoplanets considered and the particle's radii, solid material is trapped in resonances. Our calculations suggest an evolutionary scenario for the dynamics of the solid material in this region with differential capture as a function of the size. Larger particles are trapped in the outermost resonances. On the other hand, particles with sizes of order of $100 \mathrm{~m}$ or larger are not affected by drag forces. The location of our resonances is for the nebula model described in (e.g.) Cuzzi et al. (1993), different nebula models produce different quantitative results.

\section{Discussion and conclusions}

The recent discovery of the Jovian-class comets (Mumma et al. 2001b; Kawakita et al. 2001), most likely formed in the 

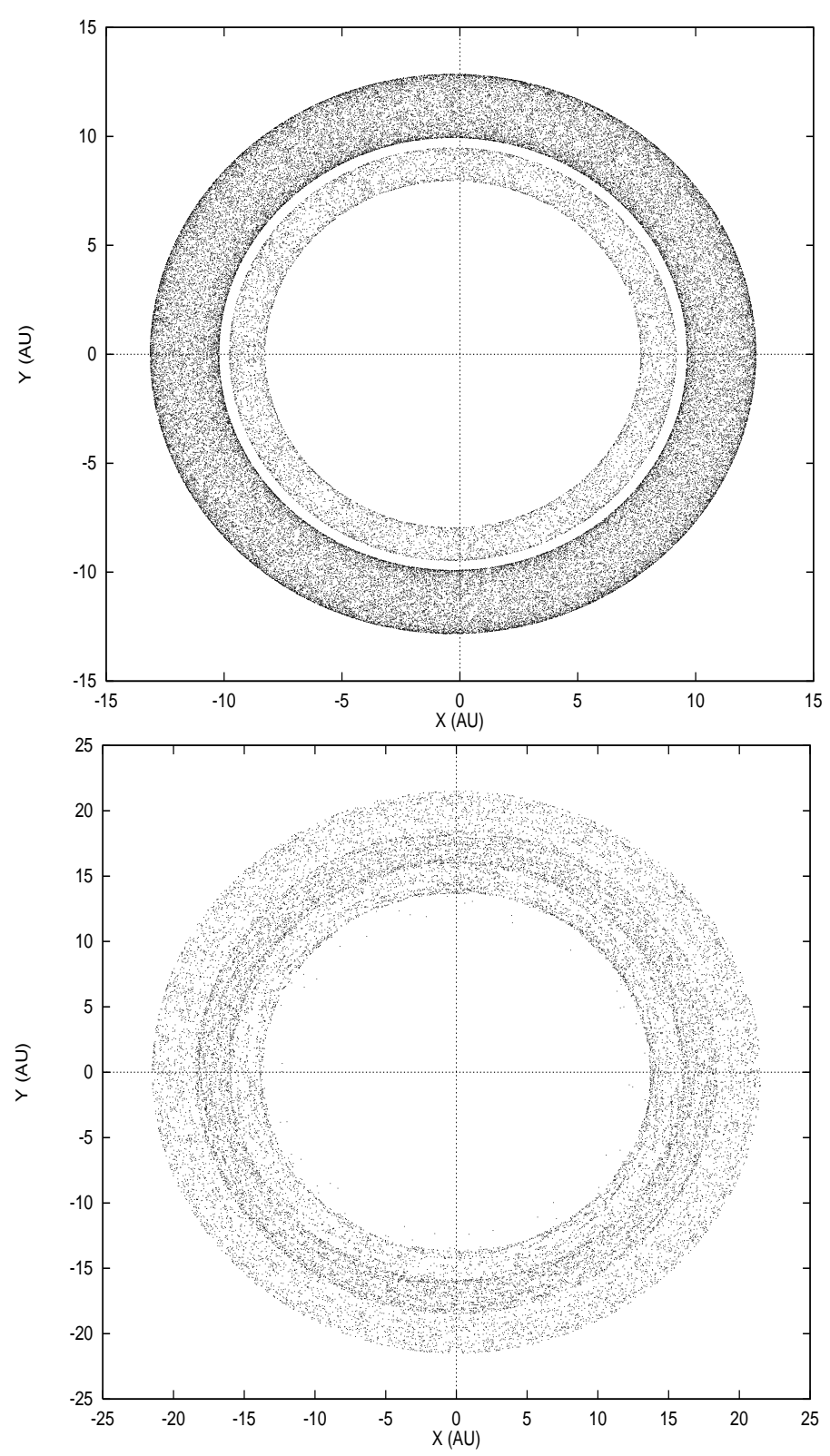

Fig. 3. Numerical simulation of the evolution of 500 metric particles under the influence of the Sun, gas drag and Jupiter (or Jupiter+Saturn). Positions of the bodies (in heliocentric Cartesian coordinates) during the trapping. Cumulative points for a time interval of $2 \mathrm{Myr}$ are plotted in order to improve contrast. (upper panel) Solid bodies subject to Sun attraction, nebular gas drag and perturbation from Jupiter. In this case the two resonances found do not overlap. (lower panel) Saturn also included. Now the resonances overlap. See text for further explanations.

Jupiter-Saturn region, raises the question of how they were formed. It has been concluded (Franklin et al. 1989; Murray \& Dermott 1999) that low-eccentricity, low-inclination orbits between Jupiter and Saturn are unlikely to survive longer than $10^{7}$ years, therefore any object formed in that region during the early stages of the formation of the outer Solar System was ejected long ago. Similar calculations for the Saturn-Uranus zone (Gladman \& Duncan 1990; Holman \& Wisdom 1993) gave an upper limit for the survival time scale in that region of $10^{8}$ years. From a dynamical point of view, the Jovian-class comets consist of objects formed in the Jupiter-Saturn region, in a time scale likely shorter than $10^{8}$ years, that were ejected towards a stable region beyond Neptune. Objects in these region are long-lived but not indefinitely stable and they eventually fall inwards crossing the Giant Planets region. The purpose of this paper has been to investigate a plausible scenario of the formation of the comet C/1999 S4 LINEAR, the first member of this class: trapping of solid material in resonances induced by gas drag. We have shown that growing protoplanets can confine a disk of metric sized dust particles. From an orbital dynamics point of view, our results suggest an evolutionary scenario with solid material being trapped at different distances from the growing protoplanets and the trapping distances gradually changing as the protoplanets keep growing. This may translate into a continuous spectrum in the physical properties of the hypothetical minor bodies formed. On the other hand, the mechanism discussed here is size-selective, with bodies of certain size preferentially trapped by certain resonances. The orbital changes due to gas drag are monotonic in nature; as the orbital energy diminishes there is a secular decrease in the semi-major axis of the bodies. The orbital decay of each particle continues until it reaches a commensurability strong enough for the resonant disturbing function to be comparable to the drag. Because of the sensitivity of the trapping mechanism to initial conditions, trapping may not necessarily take place at the first such resonance each body encounters. The body may well continue its orbital decay until it reaches another strong resonance, where conditions for equilibrium are favourable. This explains the gaps in size found in Fig. 4. In principle, if these bodies were the building blocks of Jovian-class comets and asteroids, some of them may be very uniform in structural composition with blocks only of a certain size contributing to the final body. It is a plausible scenario when the growing protoJupiter was the only planet present in the outer Solar System. This is, however, unlikely as the resonances overlap when two planets are considered (see Figs. 3 and 4) and fragments of different size may appear after collisions.

Very recently, Wurm et al. (2001) have pointed out that current models of km-sized planetary building blocks, or planetesimals, by collisional accretion require unrealistically low collision velocities or ad hoc assumptions about sticking in order for growth to occur. Once $\mathrm{cm}$-sized agglomerates have formed, collision velocities for typical nebula models increase by orders of magnitude to several tens of $\mathrm{m} / \mathrm{s}$ (Weidenschilling \& Cuzzi 1993). Therefore the impact velocities are so high that the smaller agglomerates should be completely disrupted and the larger bodies should be eroded or cratered. However, our results suggest that if the scenario presented in our paper is true, the collisional velocities for bodies trapped in a gas-induced resonance could be much lower, in fact lower than a few $\mathrm{m} / \mathrm{s}$. This is particularly favourable for the Jupiter-alone case where resonant rings do not overlap. As the relative velocity, $V_{\mathrm{r}}$, in a gas-induced resonance is low the total impact energy in a twobody collision

$E=\frac{1}{2} \frac{m_{1} m_{2}}{m_{1}+m_{2}} V_{\mathrm{r}}^{2}$, 

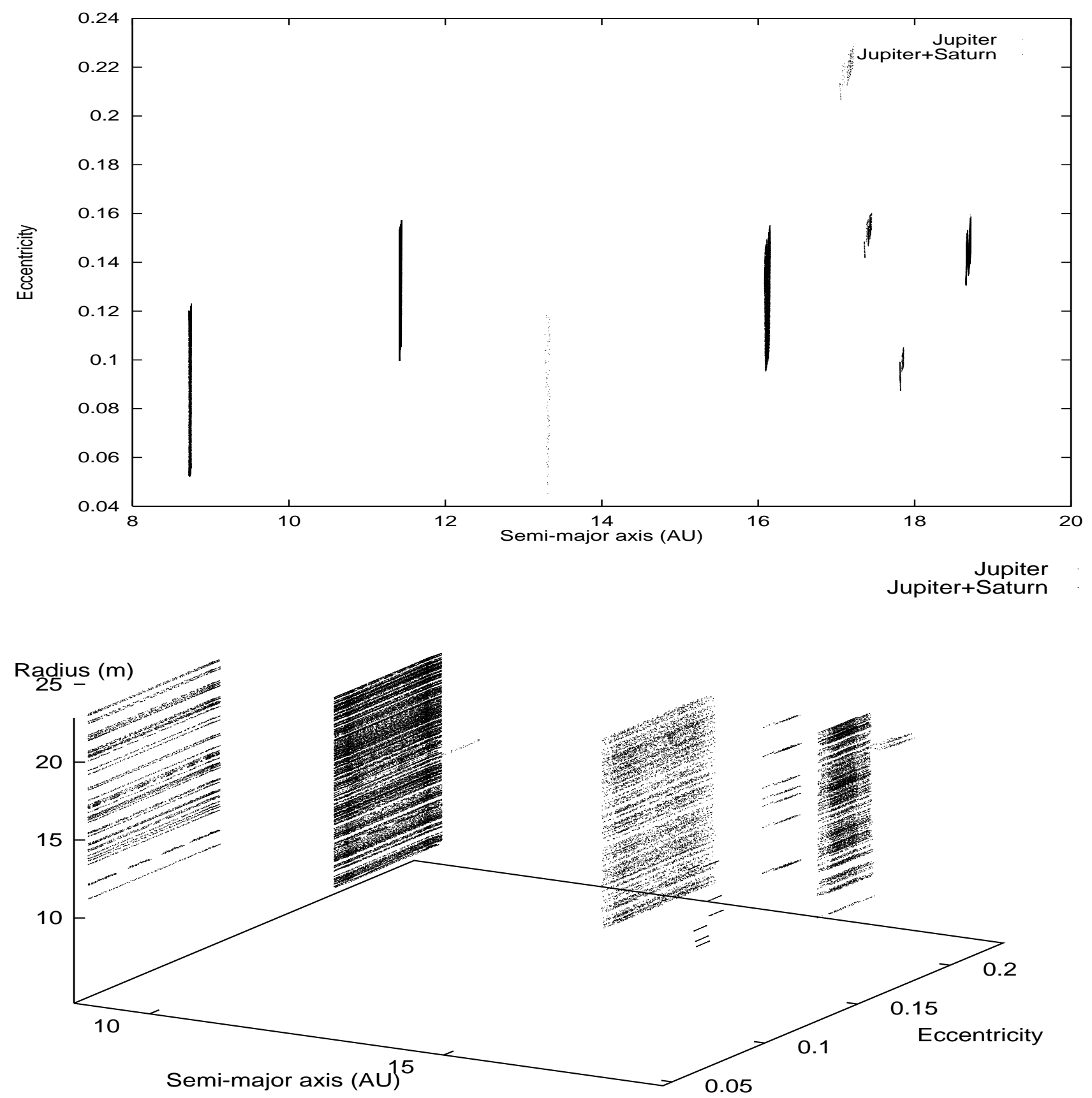

Fig. 4. Same calculations as in Fig. 3. The figures show the orbital elements (semi-major axis and eccentricity) of the particles after they are captured in the gas-induced resonances. (upper panel) Eccentricity as a function of time of the trapped particles for two different simulations, (Sun+Jupiter and Sun+Jupiter+Saturn). The two resonances found in the Jupiter-alone case shift outward when Saturn is also included. Note the additional resonances that appear when both Jupiter and Saturn are considered. (lower panel) Radius of the particle as a function of the orbital elements. The gas-induced resonant trapping is rather selective as regards particle size. See text for further explanations.

where $m_{1}$ and $m_{2}$ are the masses of the colliding bodies, is also low, making smooth sticking possible. The relative velocity is $V_{\mathrm{r}} \sim 0.5 G\left(M / a^{3}\right)^{1 / 2} \Delta a$ for two bodies separated by $\Delta a=a_{2}-a_{1}$, where $M$ is the mass of the Sun, and $G$ is the gravitational constant. In a typical resonance presented in this paper $\Delta a$ is very negligible therefore collisions are more similar to actual mergers than impacts, at least in the single planet case, where resonances do not overlap. This environment is unique in producing weakly gravitationally bound cometesimal aggregates. As heat dissipation in such an encounter is very negligible, the physicochemical structure of the building blocks could remain virtually unaltered, preserving protosolar abundances. For the single planet configuration, building blocks of the cometesimal objects are very uniform in size. If two planets are considered, then a relatively wide size spectrum is expected as collisions between bodies located in overlapping resonances may occur. The size spectrum found in C/1999 S4 LINEAR suggests that this comet was formed beyond Saturn when proto-Saturn was already present in the Solar System and therefore it may not be a genuine representative 
of the very primordial generation of minor bodies that formed soon after Jupiter. However, according to the recent constraints on the time scales of the formation of the giant planets presented by Hersant et al. (2001) and based on D/H measurements in the Solar System, the comet C/1999 S4 LINEAR may have formed when the Solar System was a few Myr old.

The results of our calculations compare well with those from Beaugé et al. (1994) although such a comparison is only possible in the Jupiter case. At the end of their collisional simulation only three bodies remain with orbital elements: $a_{1}=8.73 \mathrm{AU}, e_{1}=0.076, a_{2}=10.03 \mathrm{AU}, e_{2}=0.083$, $a_{3}=10.91 \mathrm{AU}, e_{3}=0.111$. In our non-collisional simulation we obtain two remaining rings, Fig. 3, with averaged orbital elements: $a_{1}=8.7 \mathrm{AU}, e_{1}=0.08, a_{2}=11.4 \mathrm{AU}, e_{2}=0.13$, in spite of using different nebula model, integration techniques and initial conditions. However, in our case the final orbits of the trapped bodies are of one type, $\sigma$-libration, although they also found corotation. This can be explained as a result of the non-collisional nature of our calculations (our particles do not grow or fragment) as well as different initial conditions (particle size).

On the other hand, it has become evident that the primordial Earth was totally degassed and that the volatile elements of the biosphere, including water vapor, were brought later by comets. The existence of these first generation of comets may have major implication on the rise of life on Earth. The low $\mathrm{D} / \mathrm{H}$ ratio in Earth's oceans could reflect the contribution of Jovian-class comets, which would have provided most of the mass (Delsemme 1998, 2000; Morbidelli et al. 2001), as the $\mathrm{D} / \mathrm{H}$ ratio in the oceans is very close to the mean value of the $\mathrm{D} / \mathrm{H}$ ratio of the water inclusions in carbonaceous chondrites (for a recent and detailed discussion of this problem and its constraints see Hersant et al. 2001). In this scenario, asteroids and the comets from the Jupiter-Saturn zone were the first water deliverers, when the Earth was less than half its present mass. A late impact phase of icy planetesimals known as "Late Heavy Bombardment" may have been triggered by the formation of Uranus and Neptune (Levison et al. 2001).

Recently, Tegler \& Romanishin (1998) have suggested the existence of two distinct populations of Centaurean objects. These authors found that some of the Centaurs have colors similar to the average colours of C-type asteroids. The other group includes some of the reddest objects known in the Solar System, similar (but redder) to D-type asteroids. However, Luu \& Jewitt (1996) found diversity of colours rather than two colour populations although their photometry is less accurate. If the bimodal color distribution is confirmed, grey Centaurs (the Chiron and Chariklo group) may have formed in the Jupiter-Saturn region within the scenario discussed in this paper and red Centaurs (the Nessus and Pholus group) may have appeared beyond Uranus and Neptune. To confirm or deny this hypothesis, $\mathrm{D} / \mathrm{H}$ ratio measurements for both families of objects should be carried out.

In principle, one can argue against our previous discussion and say that our present work considers the possibility that the building blocks of the unusual comet C/1999 S4 LINEAR formed in the Uranus-Neptune region, migrated inward due to gas drag, were captured into an exterior mean motion resonance with the growing proto-Jupiter and/or proto-Saturn and therefore there is somewhat of a disconnect between the calculations and the conclusions of this study as it is conceivable that the cometesimals captured in a resonance would undergo enough heating to be consistent with the chemistry discussed by Mumma et al. (2001b) but this is not discussed in the paper. However, this apparent inconsistency can be easily explained by considering in a more detailed way the dynamical behaviour of infalling material during the early stages of the formation of the outer Solar System. The survival time scale for centimetric to metric size particles in the Jupiter-Saturn region before the formation of the Giant Planets is less than a few thousand years (e.g. Weidenschilling 1977; Cuzzi et al. 1993; de la Fuente Marcos \& Barge 2001), therefore the solid material must be continuously replenished with particles from the outermost regions of the primordial nebula. Taking this fact into consideration all the solid material considered in our model has not been formed in the Uranus-Neptune but beyond that region. We choose $20 \mathrm{AU}$ as initial condition to speed up the calculations but the solid material was likely formed beyond that distance. This choice does not affect any of our conclusions. On the other hand, the papers by Mumma et al. (2001b) and Kawakita et al. (2001) are based in the spectroscopic analysis of the organic volatile composition and the $\mathrm{NH}_{2}$ respectively of C/1999 S4 LINEAR. In both cases, they are not providing any information about the chemical composition of the solid (non-volatile) component of the building blocks of the comet, to do so physical sampling is needed. When a solid particle coming from the outer regions of the primordial nebula spirals inwards as a result of gas drag, its surface is likely to be covered by ices that become depleted as the particle follows its path towards the proto-Sun. When the particle becomes trapped in one of the suggested resonances and is reprocessed by collisional coagulation to be part of a larger body some heating of the material is expected that can eventually produce chemical anomalies but local volatiles can also be trapped during the event. The surface of the newly formed body is in some way new and its composition is likely very different from the primordial of the smaller building blocks. The reprocessing has taken place at a temperature of about $200 \mathrm{~K}$. As Jupiter and Saturn are being formed, the physical environment surrounding the trapping regions is gas rich and the ices condensed from locally processed nebular gas should reflect the local composition, not the primordial one (for recent works on this topic see (e.g.) Rodgers \& Charnley 2002; Charnley \& Rodgers 2002). Later, when the newly formed object becomes dynamically unstable and is ejected outwards, the modified volatile composition is preserved under new frozen material. Thousands of million years later, when the cometary object begins its journey back to the Giant Planets region the process is inverted and the outermost frozen material is depleted as the temperature increases making the inner (and older) layers available for spectroscopic study.

Our results and discussion are not meant to provide a unique or complete model of the formation of Jovian-class objects. Instead we simply point out a plausible scenario for the formation of these objects. If, as proposed in this paper, the Jovian-class objects (and possibly some Centaurs and irregular 
satellites of Jupiter and Saturn) are the debris left by Jupiter and Saturn, the study of this new cometary population will increasingly yield new and unique insights into the conditions that existed in the solar nebula at the formation epoch of Jupiter and Saturn.

Acknowledgements. We thank Dr. C. Beaugé and Dr. S. J. Aarseth for valuable discussions about the theoretical and numerical details of their work. We thank the Department of Astrophysics of Universidad Complutense of Madrid for providing excellent computing facilities at the Centro de Proceso de Datos in Moncloa. Part of the computations described in this paper have been performed on the SGI Origin 2000 of the "Centro de Supercomputación Complutense" through the UCM project "Dinámica Estelar y Sistemas Planetarios" (CIP 454). We would like to thank the referee, L. Dones. His suggestions have lead to a substantially improved paper. In preparation of this paper, we made use of the NASA Astrophysics Data System and the ASTRO-PH e-print server.

\section{References}

Adachi, I., Hayashi, C., \& Nakazawa, K. 1976, Prog. Theor. Phys., 56, 1756

Barge, P., \& Sommeria, J. 1995, A\&A, 295, L1

Beaugé, C., \& Ferraz-Mello, S. 1993, Icarus, 103, 301

Beaugé, C., Aarseth, S. J., \& Ferraz-Mello, S. 1994, MNRAS, 270, 21

Bockelée-Morvan, D., Biver, N., Moreno, R., et al. 2001, Science, 292, 1339

Bulirsch, R., \& Stoer, J. 1966, Numer. Math., 8, 1

Charnley, S. B., \& Rodgers, S. D. 2002, ApJ, 569, L133

Cuzzi, J. N., Dobrovolskis, A. R., \& Champney, J. M. 1993, Icarus, 106,102

Delsemme, A. H. 1998, Planet. Space Sci., 47, 125

Delsemme, A. H. 2000, Icarus, 146, 313

Duncan, M., Quinn, T., \& Tremaine, S. 1987, AJ, 94, 1330

Farnham, T. L., Schleicher, D. G., Woodney, L. M., et al. 2001, Science, 292, 1348

Fernández, J. A., Tancredi, G., Rickman, H., \& Licandro, J. 1999, A\&A, 352, 327

Franklin, F., Lecar, M., \& Soper, P. 1989, Icarus, 79, 223

de la Fuente Marcos, C., \& Barge, P. 2001, MNRAS, 323, 601

de la Fuente Marcos, C., \& de la Fuente Marcos, R. 1998, EM\&P, 81, 145

de la Fuente Marcos, C., \& de la Fuente Marcos, R. 2001, A\&A, 371, 1097 de la Fuente Marcos, C., Barge, P., \& de la Fuente Marcos, R. 2002, J. Comput. Phys., 176, 276

Gladman, B. J., \& Duncan, M. J. 1990, AJ, 100, 1680

Godon, P., \& Livio, M. 2000, ApJ, 537, 396

Hersant, F., Gautier, D., \& Huré, J.-M. 2001, ApJ, 554, 391

Holman, M., \& Wisdom, J. 1993, AJ, 105, 1987

Irvine, W. M., Schloerb, F. P., Crovisier, J., Fegley, B. Jr., \& Mumma, M. J. 2000, in Protostars \& Planets IV, ed. V. Mannings, A. P. Boss, \& S. S. Russell (The University of Arizona Press, Tucson), 1159

Kary, D. M., Lissauer, J. J., \& Greenzweig, Y. 1993, Icarus, 106, 288

Kawakita, H., Watanabe, J., Ando, H., et al. 2001, Science, 294, 1089

Lecar, M., Franklin, F., \& Soper, P. 1992, Icarus, 96, 234

Levison, H. F., Dones, L., \& Chapman, C. R., et al. 2001, Icarus, 151, 286

Luu, J., \& Jewitt, D. 1996, AJ, 112, 2310

Mäkinen, J. T. T., Bertaux, J.-L., Combi, M. R., \& Quémerais, E. 2001, Science, 292, 1326

Mizuno, H. 1980, Prog. Theor. Phys., 64, 544

Morbidelli, A., Petit, J.-M., Gladman, B., \& Chambers, J. 2001, Meteoritics Planet. Sci., 36, 371

Mumma, M. J., Weissman, P. R., \& Stern, S. A. 1993, in Protostars \& Planets III, ed. E. H. Levy, J. L. Lunine, \& M. S. Matthews (The University of Arizona Press, Tucson), 1177

Mumma, M. J., et al. 2001a, ApJ, 546, 1183

Mumma, M. J., Dello Russo, N., DiSanti, M. A., et al. 2001b, Science, 292,1334

Murray, C. D., \& Dermott, S. F. 1999, Solar System Dynamics (Cambridge University Press, Cambridge)

Nurmi, P., Valtonen, M. J., Zheng, J. Q., \& Rickman, H. 2002, MNRAS, 333, 835

Patterson, C. W. 1987, Icarus, 70, 319

Rodgers, S. D., \& Charnley, S. B. 2002, MNRAS, 330, 660

Soderblom, L. A., Becker, T. L., Bennett, G., et al. 2002, Science, 296, 1087

Scholl, H., Roques, F., \& Sicardy, B. 1993, Cel. Mech. Dyn. Astron., 56,381

Stern, S. A., \& Weissman, P. R. 2001, Nature, 409, 589

Tegler, S. C., \& Romanishin, W. 1998, Nature, 392, 49

Weaver, H. A., Sekanina, Z., Toth, I., et al. 2001, Science, 292, 1329

Weidenschilling, S. J. 1977, MNRAS, 180, 57

Weidenschilling, S. J. 1994, Nature, 368, 721

Weidenschilling, S. J., \& Cuzzi, J. N. 1993, in Protostars \& Planets III, ed. E. H. Levy, J. L. Lunine, \& M. S. Matthews (The University of Arizona Press, Tucson), 1031

Weidenschilling, S. J., \& Davis, D. R. 1985, Icarus, 62, 16

Wurm, G., Blum, J., \& Colwell, J. E. 2001, Icarus, 151, 318 